\title{
Factors Affecting the Evolution of Chinese Elderly Depression: A Cross-Sectional Study
}

\author{
Longyan Cui \\ College of Public Health, Dalian Medical University \\ ding Ding ( $\square$ dingdingmail0000@126.com ) \\ College of Public Health, Dalian Medical University \\ Junfeng Chen \\ College of Public Health, Dalian Medical University \\ Minghui Wang \\ College of Public Health, Dalian Medical University

\section{Fanrong $\mathrm{He}$} \\ College of Public Health, Dalian Medical University \\ Shiyang Yu \\ Dalian Municipal Center of Disease prevention and control
}

\section{Research Article}

Keywords: Depression, Chinese elder, CHARLS, Tendency

Posted Date: July 28th, 2021

DOI: https://doi.org/10.21203/rs.3.rs-707029/v1

License: (1) (1) This work is licensed under a Creative Commons Attribution 4.0 International License. Read Full License

Version of Record: A version of this preprint was published at BMC Geriatrics on February 8th, 2022. See the published version at https://doi.org/10.1186/s12877-021-02675-z. 


\section{Abstract}

Background: In the past decades, China's rapid economic growth has been accompanied by rapid changes in lifestyle and an increasing prevalence of mental disorders. This study explored the changes and factors associated with depression among the elderly population of China from 2011-2018.

Method: Data were obtained from the China Health and Retirement Longitudinal Study. The total sample size consists of 21,034 individuals aged $\geq 60$ years, and the sample sizes in 2011, 2013, 2015 and 2018 were 5081, 4853, 5027, 6343, respectively. Depressive symptoms were measured by the 10-item Short-Form developed by the Center for Epidemiological Studies Depression.

Result: We found that the tendency in depression rate of the Chinese elderly from 2011 (36.8\%) to 2018 (44.5\%). The results showed poor health $(\mathrm{OR}=3.553)$, respondents in $2018(\mathrm{OR}=2.213)$, $A D L$ damage $(\mathrm{OR}=2.010)$, multiple chronic diseases $(\mathrm{OR}=1.287)$, and western $(\mathrm{OR}=1.777)$ are risk factors for depression.

Conclusion: The rate of depression of the elderly people in China has risen dramatically. Therefore, additional steps to prevent, treat and care for the affected population are needed, Mental health prevention and treatment strategies should be incorporated into China's public health policies in a timely manner to mitigate the serious economic burden caused by the increase of depression.

\section{Introduction}

Depression is defined as a multi-dimensional heterogeneous condition, that has a negative impact on the individual's physical health, quality of life and psychosocial functioning, and may lead to disability and death[1]. Genetic dispositions, health behaviors, physical exercise, adequate sleep, chronic diseases and social support are related to depression according to considerable research[2-5], but the specific pathogenesis of depression is still being explored[6].

Nowadays, Depression has become the second major risk factor affecting people's health. Approximately 350 million people worldwide suffer from depression [7]. The global economic burden of diseases caused by depression is one of the top five among all types of diseases[8]. It accounts for $4 \%$ of the global burden of disease [6] and the global economic burden of non-fatal diseases of 10\% [9]. The difference in the prevalence of depression is caused by regional differences. In high-income countries, such as the proportion of elderly depression is $6.6 \%$ (the United States) [10] and 3.7\% (Singapore)[11]. In contrast, the incidence of depression in the elderly in low-and middle-income countries is higher[12]. For example, the prevalence of depression in the elderly over 60 years old is $9 \%$ (African)[8] and 7.8-34.8\% (Asia)[13]. The challenge of depression in the elderly in developing countries is even more serious.

China is transitioning from a developing to developed country with a large, aging population, the situation of depression among in the elderly is particularly acute. In recent years, the detection rate of depression in the elderly has reached $33 \%$ of the total population[14]. On average, about $\$ 42.67$ per capita annual medical expense is associated with treatment of symptoms and depression in China[15]. Mental health status has significant effects on individual medical expenses[16]. There are more than 264 million people over the age of 60 in China in 2020[17], and it is estimated that by 2050, the elderly population will increase to 480 million[7]. In this sense, it's necessary to carry out research on the depression of the elderly in China.

Most of the existing studies on depression in the elderly have explored the current prevalence of depression in the elderly on the basis of cross-sectional research $[2,7,18]$. However, the socio-economic status and disease spectrum have also undergone tremendous changes, which may be inferred the influencing factors and forms of the disease change[19,20]. Therefore, the main goals of this study are: first, to explore the trend of changes in the rate of depression; second, to explore the effects of influencing factors on depression in the elderly over time; and finally, this research not only provides reference for other developing countries to prevent depression in the elderly, but also can provide advice for countries with severe aging.

\section{Method}

\subsection{Data sources and samples}

The data of this study were derived from the China Elderly Care and Health Needs Tracking Service Survey Database (CHARLS), a survey of the health-related conditions of the elderly aged 45 and above chaired by Professor Zhao Yao hui of Peking University[21]. The investigation has officially started in 2011 and has been revisited every two years. The fourth phase of data has been released[22]. This study excluded the following types of investigators: samples under the age of 60; missing scores for depression; missing health-related conditions, such as ADL scores, and health self-assessments, and the total sample size of the study was 21,424 .

Page 2/15 


\subsection{Study variables}

\subsubsection{Outcome variable: Depressive symptoms}

Depressive symptom was measured through a 10-item Brief Epidemiological Research Center Depression (CES-D)[23]. Existing studies have shown that the scale is highly effective and reliability[24], and it also has good validity and reliability in Chinese elderly[25]. The scale's score is between 0 and 30 by using the four-point Likert scoring method. In addition, there were two key items of reverse score in this scale. With reference to previous studies using CHARLS data, we set a critical value of 10 points or higher as the Cut-off points[26]. Therefore, depressive symptoms were divided into binary variables ( $0=$ no depressive symptoms; $1=$ depressive symptoms).

\subsubsection{Independent variable}

The core view of most contemporary depression theories is that stress can increase the risk of depression by stimulating individual cognition and possible biological processes. Relevant studies have also shown that major stressful life events are powerful factors that make depression, especially those involving social rejection, which increase the possibility of major depression[27-29].Thus beyond the socio-demographic, variables such as ADL, chronic diseases, social activities, and Nighttime sleep were included in the research.

The socio-demographic variables considered in the analysis were age $(60-69,70-79, \geq 80)$, gender (male/female) and marital status (married or unmarried), residence(urban, rural), region (East/Middle/West). The socio-economic status was determined as the educational level (elementary school and below, junior high school, high school or above). Individual annual income (RMB) were divided into " $\leq 10000 "$, "10000-20000" and " $\geq 20000 "[7,18]$.

Social activities were represented as $0=$ no social activities, $1=$ participants who participated in at least one social activity. Nighttime sleep were defined as $1=<7 \mathrm{~h}, 2=\geq 7 \mathrm{~h}[30]$. ADL disability (lack of the ability to carry out daily activities) was described as difficulties related to the following six items: dressing, bathing, eating, going to or getting out of bed, going to the toilet, and abstinence. If they cannot complete one of them independently, they were judged to be disabled ( 0 was defined as $=$ not disabled, $1=$ disabled). Participants first performed a five-level assessment of their self-reported health status (very good, very good, fair, poor, and very poor). Based on this report, "very good" or "good" was defined as "good"; "fair"; "poor" or "very bad" was expressed as "poor". Chronic diseases were divided into two groups: 1 = no disease, 2 = one or more chronic diseases.)[7].

\subsection{Statistical analysis}

All statistical analyses were performed using SPSS25. The data was expressed as a percentage of the classification value. $\chi 2$ (chi-square) analysis was used to compare whether there are differences in the prevalence of depressive symptoms in four waves; on the basis of univariate analysis, binary logistic regression analysis was used to determine the potential risk factors of depressive symptoms. Data were expressed as $\mathrm{OR}$ and $95 \% \mathrm{Cl}$. The test level was 0.05 , and the two-way p-value $\leq 0.05$ was considered statistically significant.

\section{Result}

\subsection{The characteristics of respondents and the detection rate of depression}

Overall, the rate of elderly people with depressive symptoms was 40.9\%. The rates of depression in 2011, 2013, 2015, and 2018 were (36.8\%), (38.7\%), (42.7\%), (44.5\%), respectively. More than half of the respondents were women $(54.1 \%) ; 61.8 \%$ were $60-69$ years old; $71 \%$ were married. The majority of the respondents had an education level of elementary school and below $(79.9 \%)$ and the annual income is below 10,000 yuan (70.9\%). The detailed distribution is shown in Table 1.

Table1.characteristics stratified by years $₫ \mathrm{n} \otimes \% \bigotimes$ 


\begin{tabular}{|c|c|c|c|c|c|}
\hline \multirow[t]{2}{*}{ variable } & Total & 2011 & 2013 & 2015 & 2018 \\
\hline & $\mathrm{IN}=\mathbf{2 1 4 8 4 \square}$ & $\square N=5081 \square$ & $\square N=4853 \square$ & $\mathrm{aN}=5207 \square$ & $\square N=6343 \square$ \\
\hline \multicolumn{6}{|l|}{ Depression } \\
\hline yes & $8794(40.9)$ & 1872(36.8) & 1876(38.7) & $2224(42.7)$ & $2822(44.5)$ \\
\hline no & 12690(59.1) & $3209(63.2)$ & 2977(61.3) & 2983(57.3) & $3521(55.5)$ \\
\hline \multicolumn{6}{|l|}{ Area } \\
\hline East & 6938(32.3) & 1636(32.2) & 1553(32.0) & 1665(32.0) & 2084(32.9) \\
\hline Middle & 7301(34) & 1698(33.4) & 1655(34.1) & 1804(34.6) & 2144(33.8) \\
\hline Western & 7245(33.7) & $1747(34.4)$ & 1645(33.9) & 1738(33.4) & 2115(33.3) \\
\hline \multicolumn{6}{|l|}{ Gender } \\
\hline male & $9857(45.9)$ & 2353(46.3) & $2249(46.3)$ & $2354(45.2)$ & $2901(45.7)$ \\
\hline female & $11627(54.1)$ & 2758(53.7) & $2604(53.7)$ & 2853(54.8) & $3442(54.3)$ \\
\hline \multicolumn{6}{|l|}{ Age } \\
\hline $60 \sim 69$ & $13274(61.8)$ & 3033(59.7) & $3052(62.9)$ & $3264(62.7)$ & 3925(61.9) \\
\hline $70 \sim 79$ & $6629(30.8)$ & 1620(31.9) & 1459(30.1) & 1590(30.5) & 1960(30.9) \\
\hline$\geq 80$ & 1581(7.4) & $428(8.4)$ & $342(7.0)$ & $353(8.8)$ & $458(7.2)$ \\
\hline \multicolumn{6}{|l|}{ Marriage } \\
\hline married & $17022(79.2)$ & $3947(77.7)$ & $3878(79.9)$ & $4117(79.1)$ & $5080(80.1)$ \\
\hline unmarried & $4462(20.8)$ & $1134(22.3)$ & $975(22.1)$ & 1090(20.9) & 1263(19.9) \\
\hline \multicolumn{6}{|l|}{ Education } \\
\hline Illiterate or Primary school & 17172(79.9) & 4241(83.4) & 3970(81.8) & 4172(80.1) & 4789(75.5) \\
\hline Middle school & $3322(15.5)$ & $664(13.1)$ & $701(14.4)$ & $8212(15.8)$ & $1136(17.9)$ \\
\hline High school or above & $990(4.6)$ & 176(3.5) & $182(3.8)$ & $214(4.1)$ & $418(6.6)$ \\
\hline \multicolumn{6}{|l|}{ Residence } \\
\hline rural & $16268(75.7)$ & $3898(76.7)$ & $3780(77.9)$ & 3993(76.7) & $4597(72.5)$ \\
\hline town & $5216(24.3)$ & 1183(23.3) & $1073(22.1)$ & $1214(23.3)$ & $1746(27.5)$ \\
\hline \multicolumn{6}{|l|}{ Individual income } \\
\hline$<10000$ & $15240(70.9)$ & $4620(91.1)$ & $2307(47.5)$ & 4478(86.0) & $3829(60.4)$ \\
\hline 10000 20000 & $3410(15.9)$ & $290(5.7)$ & $1700(35.1)$ & 193(3.7) & $1227(19.3)$ \\
\hline$\geq 20000$ & 2834(13.2) & $165(3.2)$ & $846(17.4)$ & $536(10.3)$ & $1287(20.3)$ \\
\hline
\end{tabular}

Table1. characteristics stratified by years $₫ \mathrm{n} \otimes \% \otimes \otimes \bigotimes$ continued $\bigotimes$ 


\begin{tabular}{|c|c|c|c|c|c|}
\hline \multirow[t]{2}{*}{ variable } & Total & 2011 & 2013 & 2015 & 2018 \\
\hline & $\mathrm{\square N}=21484 \square$ & $\square N=50810$ & $\square N=4853 \square$ & $\square N=5207 \square$ & $\square N=6343 \square$ \\
\hline \multicolumn{6}{|l|}{ Activity } \\
\hline yes & 10697(49.8) & $2361(46.5)$ & $2651(54.6)$ & $2583(50.4)$ & 3102(48.9) \\
\hline no & $10787(50.2)$ & $2720(53.5)$ & $2202(45.4)$ & $2624(49.6)$ & $3241(51.1)$ \\
\hline \multicolumn{6}{|c|}{ Nighttime sleep } \\
\hline$<7 \mathrm{~h}$ & 12848(59.8) & 2957(58.2) & $3020(62.2)$ & $3006(57.7)$ & $3865(60.9)$ \\
\hline$\geq 7 \mathrm{~h}$ & $8636(40.2)$ & 2124(41.8) & 1833(37.8) & 2201(42.3) & 2478(39.1) \\
\hline \multicolumn{6}{|c|}{ ADL disability } \\
\hline yes & 2076(9.3) & $538(10.6)$ & $424(8.7)$ & $531(10.2)$ & $583(9.2)$ \\
\hline no & 19408(90.7) & $4543(89.4)$ & $4429(91.3)$ & $4676(89.8)$ & $5760(90.2)$ \\
\hline \multicolumn{6}{|c|}{ Self-reported heath } \\
\hline good & 2716(12.6) & $452(8.9)$ & $591(12.2)$ & $652(12.5)$ & 1021(16.1) \\
\hline general & 7774(36.2) & 1378(27.1) & 1622(33.4) & 1711(32.9) & $3063(48.3)$ \\
\hline poor & 10994(51.2) & $3251(64.0)$ & $2640(54.4)$ & $2844(54.6)$ & $2259(35.6)$ \\
\hline \multicolumn{6}{|c|}{ Chronic disease } \\
\hline 0 & $6215(28.9)$ & 1005(19.8) & $1134(23.4)$ & $965(18.5)$ & $3111(49.0)$ \\
\hline$\geq 1$ & $15269(71.1)$ & $4076(80.2)$ & $3719(76.6)$ & $4242(81.5)$ & $3232(51.0)$ \\
\hline
\end{tabular}

The rate of depression in the elderly has shown an increasing trend from 2011 to 2018 , and the difference is significant $(\chi 2=85.56$, $\mathrm{P}<0.05)$. Among the newly selected samples, the detection rates of newly-added elderly people with depressive symptoms in 2013,2015 , and 2018 were (37.7\%), (40.8\%), (41.1\%), respectively. In addition, from 2011 to 2018, the rates of depressive symptoms of the elderly who have been in this survey team were (37.4\%), (40.8\%), (45.5\%), (49.8\%). The detection rate of depressive symptoms in the elderly showed an upward trend $(P<0.05)$. (insert Table 2$)$

Table 2. Chi-square analysis of detection rate of different types of elderly depression

\begin{tabular}{|c|c|c|c|c|c|c|c|c|c|}
\hline variable & \multicolumn{3}{|c|}{ Add sample ${ }^{a}$} & Trend & \multicolumn{4}{|c|}{ Duplicate sample b } & Trend \\
\hline \multicolumn{10}{|c|}{ depressed } \\
\hline Yes & $669(37.7)$ & $837(40.8)$ & $1309(41.1)$ & $\uparrow^{*}$ & $494(37.4)$ & $538(40.8)$ & $594(45.0)$ & $657(49.8)$ & $\uparrow^{\star * \star}$ \\
\hline
\end{tabular}

Notes: $\uparrow$ increasing trend; $\downarrow$ decreasing trend $d{ }^{*} P<0.05 ;{ }^{* *} \mathrm{P}<0.01 ;{ }^{* \star *} \mathrm{P}<0.001$.

aNew samples added during interval years.

${ }^{b}$ The elderly who have been in these four surveys.

\subsection{Analysis of related factors of depression}

Chi-square analysis was used to analyze the detection rate of depressive symptoms in the elderly with different characteristics. The results showed that there were differences in the rate of depression in the elderly with different chronic diseases, ADL status, self-reported health, social activities, sleep time and other characteristics $(P<0.05)$. The rate of depressive symptoms in the elderly in the western region, female, 60-69 years old, elementary school education and below, unmarried, suffering from a variety of chronic diseases, ADL disability, and poor 
health is even higher. However, it is worth noting that in the chi-square analysis in 2018, it was found that there was no difference in the rate of depressive symptoms among elderly people of different ages ( $P=0.123)$ (insert Table 3 here).

Table 3. Chi-square analysis of depression in the elderly with different characteristics

\begin{tabular}{|c|c|c|c|c|c|c|c|c|c|c|}
\hline \multirow[t]{2}{*}{ variable } & \multirow{2}{*}{$\begin{array}{l}\text { All } \\
\text { depressed }\end{array}$} & \multirow{2}{*}{$\begin{array}{l}P \text { - } \\
\text { value }\end{array}$} & \multirow{2}{*}{$\begin{array}{l}2011 \\
\text { depressed }\end{array}$} & \multirow{2}{*}{$\begin{array}{l}P \text { - } \\
\text { value }\end{array}$} & \multirow{2}{*}{$\begin{array}{l}2013 \\
\text { depressed }\end{array}$} & \multirow{2}{*}{$\begin{array}{l}\text { P- } \\
\text { value }\end{array}$} & \multirow{2}{*}{$\begin{array}{l}2015 \\
\text { depressed }\end{array}$} & \multirow{2}{*}{$\begin{array}{l}P \text { - } \\
\text { value }\end{array}$} & \multirow{2}{*}{$\begin{array}{l}2018 \\
\text { depressed }\end{array}$} & \multirow{2}{*}{$\begin{array}{l}\text { P- } \\
\text { value }\end{array}$} \\
\hline & & & & & & & & & & \\
\hline \multicolumn{11}{|l|}{ Area } \\
\hline East & $2261(25.7)$ & 0.000 & $461(24.6)$ & 0.000 & $486(25.9)$ & 0.000 & $564(25.4)$ & 0.000 & $750(26.6)$ & 0.000 \\
\hline Middle & $3026(34.4)$ & & 631(33.7) & & $622(33.2)$ & & 792(35.6) & & $981(34.8)$ & \\
\hline Western & $3507(39.9)$ & & $780(41.7)$ & & $768(40.9)$ & & 868(39.0) & & 1091(38.6) & \\
\hline \multicolumn{11}{|l|}{ Gender } \\
\hline male & 3402(38.7) & 0.000 & $713(38.1)$ & 0.000 & 735(39.2) & 0.000 & $847(38.1)$ & 0.000 & 1107(39.2) & 0.000 \\
\hline female & 5392(61.3) & & 1159(61.9) & & 1141(60.8) & & 1137(61.9) & & 1715(60.8) & \\
\hline \multicolumn{11}{|l|}{ Age } \\
\hline $60 \rrbracket 69$ & $5597(63.6)$ & 0.000 & $1154(61.6)$ & 0.023 & 1233(65.7) & 0.004 & $1455(65.4)$ & 0.000 & $1755(62.2)$ & 0.123 \\
\hline $70 \otimes 79$ & $2600(29.6)$ & & $553(29.5)$ & & $515(27.5)$ & & $648(29.1)$ & & 884(31.3) & \\
\hline$\geq 80$ & $597(6.8)$ & & 165(8.8) & & $128(6.8)$ & & $121(5.4)$ & & $183(6.5)$ & \\
\hline \multicolumn{11}{|l|}{ Marital } \\
\hline married & $6708(76.3)$ & 0.000 & $1392(74.4)$ & 0.000 & $1452(77.4)$ & 0.001 & $1690(76.0)$ & 0.000 & $2174(77.0)$ & 0.000 \\
\hline unmarried & 2086(23.7) & & $480(25.6)$ & & $424(22.6)$ & & $534(24.0)$ & & $648(23.0)$ & \\
\hline \multicolumn{11}{|l|}{ Education } \\
\hline $\begin{array}{l}\text { Illiterate or } \\
\text { Primary } \\
\text { school }\end{array}$ & 7505(85.3) & 0.000 & 1672(89.3) & 0.000 & 1615(86.1) & 0.000 & 1907(85.8) & 0.000 & 2311(81.9) & 0.000 \\
\hline Middle school & $1024(11.7)$ & & $168(9.0)$ & & 205(10.9) & & 259(11.6) & & 392(13.9) & \\
\hline $\begin{array}{l}\text { High school } \\
\text { or above }\end{array}$ & $265(3.0)$ & & $32(1.7)$ & & $56(3)$ & & $58(2.6)$ & & $119(4.2)$ & \\
\hline \multicolumn{11}{|l|}{ Residence } \\
\hline rural & $7225(82.2)$ & 0.000 & $1576(84.2)$ & 0.000 & $1587(84.6)$ & 0.000 & 1838(82.6) & 0.000 & $2224(78.8)$ & 0.000 \\
\hline town & 1569(17.8) & & $296(15.8)$ & & $289(15.4)$ & & $386(17.4)$ & & $598(21.2)$ & \\
\hline \multicolumn{11}{|l|}{$\begin{array}{l}\text { Individual } \\
\text { income }\end{array}$} \\
\hline$<10000$ & $6637(75.5)$ & 0.000 & $1759(94.0)$ & 0.000 & $958(51.1)$ & 0.000 & $2024(91.0)$ & 0.000 & $1896(67.2)$ & 0.000 \\
\hline 10000 20000 & $1406(16.0)$ & & $88(4.7)$ & & 698(37.2) & & $65(2.9)$ & & $555(19.7)$ & \\
\hline$\geq 20000$ & $751(8.5)$ & & $25(1.3)$ & & $220(11.7)$ & & $135(6.1)$ & & $371(13.1)$ & \\
\hline \multicolumn{11}{|l|}{ Activity } \\
\hline yes & $3970(45.1)$ & 0.000 & $757(40.1)$ & 0.000 & $932(49.7)$ & 0.000 & $1003(45.1)$ & 0.000 & $1278(45.3)$ & 0.000 \\
\hline no & 4824(54.9) & & $1115(59.6)$ & & $944(50.3)$ & & 1221(54.9) & & $1544(54.7)$ & \\
\hline \multicolumn{11}{|l|}{$\begin{array}{l}\text { Nighttime } \\
\text { sleep }\end{array}$} \\
\hline$<7 h$ & $6078(69.1)$ & 0.000 & 1278(68.3) & 0.000 & $1370(73.0)$ & 0.000 & 1463(65.8) & 0.000 & $1967(69.7)$ & 0.000 \\
\hline$\geq 7 \mathrm{~h}$ & 2716(30.9) & & 594(31.7) & & $506(27.0)$ & & $761(34.2)$ & & $855(30.3)$ & \\
\hline
\end{tabular}


Notes: *P<0.05; **P<0.01;***P<0.001.

Table3. Chi-square analysis of depression in the elderly with different characteristics(continued)

\begin{tabular}{|c|c|c|c|c|c|c|c|c|c|c|}
\hline \multirow[t]{2}{*}{ variable } & \multirow{2}{*}{$\begin{array}{l}\text { All } \\
\text { depressed }\end{array}$} & \multirow{2}{*}{$\begin{array}{l}\text { P- } \\
\text { value }\end{array}$} & \multirow{2}{*}{$\begin{array}{l}2011 \\
\text { depressed }\end{array}$} & \multirow{2}{*}{$\begin{array}{l}\mathrm{P} \text { - } \\
\text { value }\end{array}$} & \multirow{2}{*}{$\begin{array}{l}2013 \\
\text { depressed }\end{array}$} & \multirow{2}{*}{$\begin{array}{l}\text { P. } \\
\text { value }\end{array}$} & \multirow{2}{*}{$\begin{array}{l}2015 \\
\text { depressed }\end{array}$} & \multirow{2}{*}{$\begin{array}{l}\mathrm{P}- \\
\text { value }\end{array}$} & \multirow{2}{*}{$\begin{array}{l}2018 \\
\text { depressed }\end{array}$} & \multirow{2}{*}{$\begin{array}{l}\mathrm{P} \text { - } \\
\text { value }\end{array}$} \\
\hline & & & & & & & & & & \\
\hline \multicolumn{11}{|c|}{ ADL disability } \\
\hline yes & $1275(14.5)$ & 0.000 & $937(50.1)$ & 0.000 & $246(13.1)$ & 0.000 & $336(15.1)$ & 0.000 & $397(14.1)$ & 0.000 \\
\hline no & 7519(85.5) & & $935(49.9)$ & & 1630(86.9) & & 1888(84.9) & & $2425(85.9)$ & \\
\hline \multicolumn{11}{|c|}{$\begin{array}{l}\text { Self-reported } \\
\text { heath }\end{array}$} \\
\hline good & $575(6.5)$ & 0.000 & 71(3.8) & 0.000 & $119(6.3)$ & 0.000 & $142(6.4)$ & 0.000 & $243(8.6)$ & 0.000 \\
\hline general & $2587(29.5)$ & & $359(19.2)$ & & $507(27.1)$ & & $581(26.1)$ & & $1140(40.4)$ & \\
\hline poor & $5632(64.0)$ & & $1442(77.0)$ & & $1250(66.6)$ & & $1501(67.5)$ & & $1439(51.0)$ & \\
\hline \multicolumn{11}{|l|}{$\begin{array}{l}\text { Chronic } \\
\text { disease }\end{array}$} \\
\hline 0 & 2172(24.7) & 0.000 & 281(15.0) & 0.000 & $349(18.6)$ & 0.000 & $312(14.0)$ & 0.000 & $1230(43.6)$ & 0.000 \\
\hline$\geq 1$ & $6622(75.3)$ & & 1591(85.0) & & 1527(81.4) & & 1912(86.0) & & $1592(56.4)$ & \\
\hline
\end{tabular}

Notes: *P<0.05; **P<0.01; ***P<0.001.

\subsection{Logistic regression model}

Regression analysis was performed for each wave, we found that the results of each year showed an association between region, gender, marriage, place of residence, sleep, social activities, self-rated health, chronic diseases, ADL and depression (Table 4). Then based on the overall regression analysis, the independent variable of year was included in the regression model 6 . The final model 6 results show that poor health (OR=3.553), ADL disability (OR=2.010), respondents in 2018 (OR=2.213), Western (OR=1.777), women (OR=1.521); multiple chronic diseases $(O R=1.287)$ and unmarried $(O R=1.2946)$ are risk factors for depression. Sleeping time at night $\geq 7(O R=0.549)$, living in town(OR=0.635), annual income $\geq 20000$ yuan(OR=0.741), and social activities $(\mathrm{OR}=0.825)$ are protective factors for depression in the elderly(insert Table 5 here).

Table 4. Binary logistic regression analysis of depressive symptoms: OR (95\% CI) 


\section{variable}

\begin{tabular}{ll} 
Model $1^{\mathrm{a}}$ & Model $2^{\mathrm{b}}$ \\
\hline OR $(95 \% \mathrm{Cl})$ & OR $(95 \% \mathrm{Cl})$
\end{tabular}

Model $3^{c}$

Model $4^{\mathrm{d}}$

OR $(95 \% \mathrm{Cl})$

OR $(95 \% \mathrm{Cl})$

OR $(95 \% \mathrm{Cl})$

Area (East)

Middle

$1.391^{\star \star \star}(1.190-$

$1.279^{\star \star} \otimes 1.095-1.494 \rrbracket$

$1.554^{\star \star \star} \otimes 1.341-$

1.802》

$1.414^{\star \star \star} \otimes 1.235$

1.626)

$1.758^{\star \star \star} \otimes 1.507-2.050 \otimes$

$1.791^{\star * *}(1.535-$

2.089ه

$1.779^{\star \star \star} \otimes 1.534-$

2.064】

1.618》

Western

$1.569^{\star \star \star} \otimes 1.380-$

1.783》

$1.502^{\star \star \star} \otimes 1.320-1.709 \rrbracket$

$1.512^{\star \star \star} \otimes 1.336-$

$1.712 \rrbracket$

$1.514^{\star \star \star} \otimes 1.351-$

female

$0.847^{*} \bowtie 0.737-0.973 \rrbracket$

$0.801^{\star \star} \bowtie 0.695-0.924 \rrbracket$

$0.771^{\star \star \star} \bowtie 0.674$

$0.881 \rrbracket$

$\geq 80$

0.933凶0.737-1.181》

$0.965 \rrbracket 0.743-1.254 \rrbracket$

$0.547^{\star \star \star} \llbracket 0.421-$

$0.711 \rrbracket$

Marital $\bigotimes$ married $\rrbracket$

unmarried

$1.233^{\star \star} \otimes 1.058$

1.439凶

$1.208^{*} \otimes 1.027-1.421 \rrbracket$

$1.440^{\star \star \star} \otimes 1.235$

$1.678 \rrbracket$

$1.740^{\star \star \star} \otimes 1.521-$

1.9908

Education\IIliterate or Primary school》

Middle school

$0.746^{\star \star} \bowtie 0.605-$ $0.920 \rrbracket$

0.846ه0.693-1.033®

$0.744^{\star \star} \rrbracket 0.621-0.891 \rrbracket$

$0.808^{\star *} \rrbracket 0.691-0.944 \rrbracket$

High school or above

$0.645^{\star} \rrbracket 0.422-0.986 \rrbracket$

1.190ه0.830-1.706ه

$0.799 \rrbracket 0.566-1.128 \rrbracket$

$0.687^{\star \star} \rrbracket 0.536-0.880 \rrbracket$

Residence\rural『

town

$$
\begin{aligned}
& 0.600^{\star \star \star} \bowtie 0.506- \\
& 0.712 \rrbracket
\end{aligned}
$$

$0.552^{\star \star \star} \bowtie 0.445-0.684 \rrbracket$

$0.646^{\star \star \star} \otimes 0.550-$
$0.760 \rrbracket$

$0.703^{\star \star \star} \llbracket 0.606-$
$0.816 \rrbracket$

Individual income $₫<10000 \rrbracket$

10000 20000

$0.856 \rrbracket 0.649-1.128 \rrbracket$

$1.014(0.884-1.164)$

$0.640 * *(0.462-$

$0.887)$

$\geq 20000$

$0.630 \star \approx 0.399-0.994 \rrbracket$

$0.889(0.693-1.138)$

$0.625^{\star \star \star}(0.494-$

$0.792)$

$0.965(0.838-1.111)$

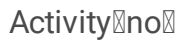

yes

$$
\begin{aligned}
& 0.796^{\star \star \star} \bowtie 0.703- \\
& 0.901 \rrbracket
\end{aligned}
$$

$0.803^{\star \star}(0.708-0.911)$

$0.795^{\star \star \star}(0.705-$
$0.897)$

Nighttime sleep $\llbracket<7 \mathrm{~h} \rrbracket$

$\geq 7 \mathrm{~h}$

$0.552^{\star \star \star} \bowtie 0.486-$

$0.480^{\star \star \star}(0.421-0.548)$

$0.581^{\star \star \star}(0.515-$
$0.656)$

0.626 《

ADL disability $₫$ no $\bigotimes$

yes

$1.931^{\star \star \star} \otimes 1.589-$

2.345》

$2.0149^{\star \star \star}(1.621-$

2.503)

$2.129^{\star \star \star}(1.744-$
$2.601)$

Self-reported heath $\nabla$ good $\nabla$

general

poor $1.758^{\star \star \star} \otimes 1.314-$

2.351区

$3.477^{\star \star \star} \bowtie 2.643$ $1.660^{\star \star \star}(1.311-2.102)$

$1.620^{\star \star \star}(1.300-$

2.018)

$2.981^{\star \star \star}(2.382-3.732)$

$3.338^{\star \star \star}(2.707-$
0.897(0.804-1.002)

$0.567^{\star \star \star}(0.507-$

$1.900^{\star \star \star}(1.561-$

$0.711^{\star \star \star}(0.595-$

0.851)

$0.635)$

2.313) 
Chronic disease $₫ 0 \rrbracket$

$$
\geq 1
$$

Notes: ${ }^{*} \mathrm{P}<0.05 ;{ }^{* \star} \mathrm{P}<0.01 ;{ }^{* \star *} \mathrm{P}<0.001$.

aThe logistic model of 2011.

bThe logistic model of 2013.

cThe logistic model of 2015.

dThe logistic model of 2018. Age was excluded in the model.

Table 5. Binary logistic regression analysis of depressive symptoms: OR (95\% CI) 


\begin{tabular}{|c|c|c|c|}
\hline \multirow[t]{2}{*}{ variable } & \multirow[t]{2}{*}{ Reference } & \multirow{2}{*}{$\begin{array}{l}\text { Model } 5^{a} \\
\text { OR }(95 \% \mathrm{Cl})\end{array}$} & \multirow{2}{*}{$\begin{array}{l}\text { Model } 6^{b} \\
\text { OR }(95 \% \mathrm{Cl})\end{array}$} \\
\hline & & & \\
\hline \multicolumn{4}{|l|}{ Area } \\
\hline Middle & East & $1.420^{\star \star \star}(1.321-1.528)$ & $1.416^{\star \star \star} \otimes 1.315-1.524 \rrbracket$ \\
\hline Western & & $1.782^{\star \star \star}(1.657-1.918 \rrbracket$ & $1.777^{\star \star \star} \bigotimes 1.652-1.912 \rrbracket$ \\
\hline \multicolumn{4}{|l|}{ Gender } \\
\hline female & male & $1.538^{\star \star \star} \oslash 1.447-1.634 \rrbracket$ & $1.521^{\star \star \star} \otimes 1.431-1.618 \rrbracket$ \\
\hline \multicolumn{4}{|l|}{ Age } \\
\hline $70 \otimes 79$ & $60 \rrbracket 69$ & $0.835^{\star \star \star} \llbracket 0.782-0.892 \rrbracket$ & $0.832^{\star \star \star} \rrbracket 0.779-0.890 \rrbracket$ \\
\hline$\geq 80$ & & $0.780^{\star \star \star} \rrbracket 0.691-0.880 \rrbracket$ & $0.798^{\star \star \star} \bowtie 0.707-0.901 \rrbracket$ \\
\hline \multicolumn{4}{|l|}{ Marital } \\
\hline unmarried & married & $1.279^{\star \star \star} \oslash 1.186-1.379 \rrbracket$ & $1.294^{\star \star \star} \nabla 1.199-1.396 \rrbracket$ \\
\hline \multicolumn{4}{|l|}{ Education } \\
\hline Middle school & Illiterate or Primary school & $0.795^{\star \star \star} \bowtie 0.726-0.870 \rrbracket$ & $0.775^{\star \star \star} \bowtie 0.708-0.849 \rrbracket$ \\
\hline High school or above & & $0.813^{*} \llbracket 0.693-0.954 \rrbracket$ & $0.771^{\star \star} \llbracket 0.656-0.907 \rrbracket$ \\
\hline \multicolumn{4}{|l|}{ Residence } \\
\hline town & rural & $0.620^{\star \star \star} \bowtie 0.571-0.673 \rrbracket$ & $0.635^{\star \star \star} \rrbracket 0.584-0.691 \rrbracket$ \\
\hline \multicolumn{4}{|l|}{ Individual income } \\
\hline $10000 \sim 20000$ & $<10000$ & $0.983 \rrbracket 0.907-1.066 \rrbracket$ & $0.923(0.845-1.007)$ \\
\hline$\geq 20000$ & & $0.858^{\star \star} \bowtie 0.770-0.957 \rrbracket$ & $0.741^{\star \star \star}(0.662-0.829)$ \\
\hline \multicolumn{4}{|l|}{ Activity } \\
\hline yes & no & $0.819^{\star \star \star} \llbracket 0.772-0.869 \rrbracket$ & $0.825^{\star \star \star}(0.777-0.875)$ \\
\hline \multicolumn{4}{|l|}{ Nighttime sleep } \\
\hline$\geq 7 \mathrm{~h}$ & $<7 \mathrm{~h}$ & $0.545^{\star \star \star} \bowtie 0.513-0.579 \rrbracket$ & $0.549^{\star \star \star}(0.517-0.584)$ \\
\hline \multicolumn{4}{|l|}{ ADL disability } \\
\hline yes & no & $2.048^{\star \star \star} \otimes 1.854-2.262 \rrbracket$ & $2.010^{\star \star \star}(1.817-2.222)$ \\
\hline \multicolumn{4}{|l|}{ Self-reported heath } \\
\hline general & good & $1.730^{\star \star \star} \otimes 1.554-1.925 \rrbracket$ & $1.711^{\star \star \star}(1.537-1.906)$ \\
\hline poor & & $3.220^{\star \star \star} \oslash 2.901-3.575 \rrbracket$ & $3.553^{\star \star \star}(3.197-3.950)$ \\
\hline \multicolumn{4}{|l|}{ Chronic disease } \\
\hline$\geq 1$ & 0 & $1.113^{\star \star}(1.041-1.190)$ & $1.287^{\star \star \star}(1.200-1.380)$ \\
\hline \multicolumn{4}{|l|}{ Years } \\
\hline 2013 & 2011 & & $1.287^{\star \star \star}(1.172-1.413)$ \\
\hline 2015 & & & $1.496^{\star \star *}(1.374-1.630)$ \\
\hline 2018 & & & $2.213^{\star \star \star}(2.025-2.419)$ \\
\hline
\end{tabular}


Notes: ${ }^{*} \mathrm{P}<0.05 ;{ }^{* \star} \mathrm{P}<0.01 ; * \star \star * P<0.001$.

aThe logistic model of total sample, excluding years.

bon the basis of model1, years was added in the model.

\section{Discussion}

The study focused on the trend of changes in the rate of depression among the elderly in China. The results show that the rate of depression in the elderly in China has reached 44.5\% (in 2018), which is higher than the results of a similar domestic study[7]. Our research also demonstrated that the rate of depressive symptoms showed an upward trend in both the newly-added elderly interviewed and the elderly who have always been in this survey cohort. This finding indicated that the increase in the detection rate of depression caused by time changes is indeed an issue in China, and steps must be taken to improve the mental health condition of elderly.

Research results show that elderly people with poor health $(O R=3.553), A D L$ impairment $(O R=2.010)$ and multiple chronic diseases $(O R=$ 1.287) have a higher risk of depression, which is consistent with previous research conclusions $[3,7,18]$. Chronic diseases will cause the health of the elderly to deteriorate, and long-term medication after illness will increase the economic burden of the disease. In addition, due to the damage of ADL, the majority of the elderly rely on the care of family members, which increases the financial burden of the family, and the elderly will also have psychological burden problems. Moreover, it is inconvenient for them to participate in social activities and this prevents them from being able to talk about their inner emotions and may exacerbate depression [31-33].

Sleep is a very important factor affecting health, which has been found to cause many diseases and even all-cause mortality[34]. Our research also indicate that elderly people who sleep for more than 7 hours $(O R=0.549)$ have a lower risk of depression,which is consistent with the study of Gehrman P[35]. Daytime physical fatigue or mental fatigue caused by poor night sleep quality may disrupt the circadian rhythm or cause hormonal changes. Furthermore, lack of sleep at night may be one of the causes of mental disorders or the sequelae of previous mental disorders, and these factors can cause depression produce[34, 36-38].

There is consensus that SES plays an important role in health[39]. Our research found that elderly people with higher personal annual income $(\mathrm{OR}=0.741)$ have a lower risk of depression. It has been suggested that poor SES may lead to poor access to mental health services, and further affect the diagnosis and treatment of depression. It is difficult for low-income populations to attend to healthcare needs and to be screened for depression symptoms[40]. Divorce $(O R=1.294)$ was found to be a risk factor for depression, which is consistent with the findings of Zhang $Y$ and Ouyang $P[7,41]$. As a special group, the elderly may rely more on social support, especially support from family members[42]. The absence of a spouse not only means that the financial support from the spouse is weakened, but also they cannot share the inner feelings with others, This may increase the possibility of depression in the divorced elderly $[7,43]$.

Social activity $(\mathrm{OR}=0.825)$ was also confirmed as a meaningful factor for depression. The review of empirical literature by Adams et al. reveals that socializing (e.g., spending time with friends, family, or neighbors) has the strongest effect on well-being in late life[44]. By comparison, infrequent participation in social activities is an indicator of social isolation[45]. What's more, events involving interpersonal stress and social rejection are the strongest risk factors for depressive symptoms[46]. Hence social activities should be promoted as an important means of preventing depression in the elderly.

In addition to the factors discussed above, we also found that the West region $(\mathrm{OR}=1.777)$, women $(\mathrm{OR}=1.521)$, higher education $(\mathrm{OR}=$ $0.771)$, and living in city $(O R=0.631)$ are associated with depression. Considerable research demonstrates that these factors are very important in terms of the potential harm associated with depression $[9,18,40,47]$. In areas with better economic conditions, the healthrelated policies, living surroundings and community infrastructure always show better conditions, which are effective in promoting mental health $[47,48]$. Experience with rejection, criticism and separation are the factors leading to depression. Women are more likely to perceive these emotions, which leads to a higher likelihood of them suffering from depression[49].

Besides, we also explored the effect of the passage of time on depression in the elderly. This study confirmed that the rate of depression among the elderly in China has increased, and the respondents in $2018(\mathrm{OR}=2.213)$ have a higher risk of depression. The rate of depressive symptoms increased by nearly 10\% from 2011 (36.8\%) to 2018 (44.5\%). By contrast, in the 1990s, the prevalence of depression among the elderly in China was about $3.86 \%$ in mainland[50]. The reasons for this phenomenon may be as follows: : First, with the rapid development of economy in China, people's material life has improved, and their average life expectancy has also increased along with advances in the availability of medical treatment. That in turn would increase the rate of depression in elderly[51].Second, with rapid socioeconomic transition and the erosion of the traditional family, a significant boom of the empty nest elderly occurred, especially in rural

Page $11 / 15$ 
areas[52], which may contribute to the morbidity of mental diseases in the Chinese elderly, especially depression[53].Third, more and more digital service technologies are applied to all aspects of people's lives, such as convenient payment and online shopping (Apart from increased convenience, modern technology may increase social estrangement). In fact, because of physical aging and diseases, the elderly, especially divorce, ADL disability, living alone, are the ones who need to rely on digital services most in society. However, limited by education, physical conditions, and acceptance, they are often trapped in these convenient digital services, resulting in the feeling of social disconnection, which can easily lead to psychological problems.

\section{Strengths and limitations}

Interpretation of these findings should take into consideration several limitations. Firstly, the data we used in this survey were crosssectional study. We cannot draw conclusions about whether there is a clear causal relationship between depressive symptoms and the factors being studied. Secondly, the depression detection scale in this study can only be used as a reference opinion, and the diagnosis requires further clinical diagnosis. Finally, there were limited considerations about the factors that affect the occurrence of depression in the elderly, and subsequent research could further explore the role of depression and other factors under the influence of time.

\section{Conclusion}

These above conclusions shed light on the urgent need for reforming the current mental health system in China, and further government involvement is required to improve the treatment and prevention of the mental health conditions. The following suggestions are put forward for the depression of the elderly: (1) The government should use the power of the social organizations to deal with the various needs of the elderly, improve the quality of life of older people. For example, the establishment of a community mental health care center for the elderly. According to the mental characteristics of the elderly, conduct individual mental health consultations, answer the mental problems encountered by the elderly in their daily lives, and help them get out of their psychological dilemmas; (2) Make full use of the various resources in the community to promote various elderly care services in the community, and provide paid family services (such as nannies and hourly labor caregivers) for the elderly who are inconvenient or difficult to live with. This would include purchasing daily necessities, sick care, providing company, etc. They might assist the elderly to establish cultural organizations and activities for the elderly according to their own interests, which enriches the spiritual life of the elderly. (3) Family members should play an irreplaceable function of spiritual support for the elderly and pay attention to the changes in the mental health of the elderly in time. As well as advocating the elderly to maintain an optimistic attitude towards life.

\section{Abbreviations}

SES: Socioeconomic Status $\mathbb{A D L}$ : Activities of Daily Living

\section{Declarations}

Ethics approval and consent to participatelThis research is based on an open database-China Health and Retirement Longitudinal Survey (CHARLS), which is hosted by the National Development Research Institute of Peking University and jointly executed by the Chinese Social Science Survey Center of Peking University and the Youth League Committee of Peking University. The large-scale interdisciplinary survey project is a major project funded by the National Natural Science Foundation of China. All participants in the project signed written informed consent.

Consent for publication: Not applicable.

Data and materials: The data used in this study is a public database released by the CHARLS project team. Longyan Cui registered the platform and obtained permission to use the data.

Competing interests: None of authors have any competing interests.

Funding: This research did not receive any specific funding.

Author's contributions: L.Y.C Mainly analyzed the data and wrote the manuscript; D.D designed the study and reviewed the paper; J.F.CH provided advice on the writing of the paper; M.H.W, SYY and F.R.HE responsible for collecting and cleansing the data.

Acknowledgements: Not applicable. 


\section{References}

1.Atkins R. Self-efficacy and the promotion of health for depressed single mothers. Ment Health Fam Med. 2010.7(3):155-68.

2.Yu J, Li J, Cuijpers P, Wu S, Wu Z. Prevalence and correlates of depressive symptoms in Chinese older adults: a population-based study. Int J Geriatr Psychiatry. 2012.27(3):305-12.

3.Alexopoulos GS. Depression in the elderly. Lancet. 2005.365(9475).

4.Zhai L, Zhang H, Zhang D. SLEEP DURATION AND DEPRESSION AMONG ADULTS: A META-ANALYSIS OF PROSPECTIVE STUDIES. Depress Anxiety. 2015.32(9):664-70.

5.Tough H, Siegrist J, Fekete C. Social relationships, mental health and wellbeing in physical disability: a systematic review. BMC Public Health. 2017.17(1):414.

6.Gałecki P, Talarowska M. The Evolutionary Theory of Depression. Med Sci Monit.

2017.23:2267-2274.

7.Zhang Y, Liu Z, Zhang L, Zhu P, Wang X, Huang Y. Association of living arrangements with depressive symptoms among older adults in China: a cross-sectional study. BMC Public Health. 2019.19(1):1017.

8.Dadi AF, Wolde HF, Baraki AG, Akalu TY. Epidemiology of antenatal depression in Africa: a systematic review and meta-analysis. $B M C$ Pregnancy Childbirth. 2020.20(1):251.

9.Salk RH, Hyde JS, Abramson LY. Gender differences in depression in representative national samples: Meta-analyses of diagnoses and symptoms. Psychol Bull. 2017.143(8):783-822.

10.Dallo FJ, Prabhakar D, Ruterbusch J, Schwartz K, Peterson EL, Liu B, Ahmedani BK. Screening and follow-up for depression among Arab Americans. Depress Anxiety. 2018.35(12):1198-1206.

11.Lau YW, Vaingankar JA, Abdin E, Shafie S, Jeyagurunathan A, Zhang Y, Magadi H, Ng LL, Chong SA, Subramaniam M. Social support network typologies and their association with dementia and depression among older adults in Singapore: a cross-sectional analysis. $B M J$ Open. 2019.9(5):e025303.

12.Gureje O. Late-life depression in socially and culturally diverse settings. Epidemiol Psychiatr Sci. 2020.29:e168.

13.Tengku Mohd TAM, Yunus RM, Hairi F, Hairi NN, Choo WY. Social support and depression among community dwelling older adults in Asia: a systematic review. BMJ Open. 2019. 9(7): p. e026667.

14.Nie XL,Wang HY, Sun F, et al. Detection rate of depression among community-dwelling older adults in China: a systematic review and updated meta-analysis of studies in 2000-2012. Chin Ment Health J. 2013;27(11):805e14.

15.Hsieh CR, Qin X. Depression hurts, depression costs: The medical spending attributable to depression and depressive symptoms in China. Health Econ.2018. 27(3): p. 525-544.

16.Sun X, Zhou M, et al. Depressive costs: medical expenditures on depression and depressive symptoms among rural elderly in China. Public Health. 2020. 181: p. 141-150.

17.www.stats.gov.cn. [accessed 25 June 2021].

18.Fang M, Mirutse $\mathrm{G}$, et al. Role of socioeconomic status and housing conditions in geriatric depression in rural China: a cross-sectional study. BMJ Open. 2019. 9(5): p. e024046.

19.Ryder AG, Sun J, et al. Depression in China: integrating developmental psychopathology and cultural-clinical psychology. J Clin Child Adolesc Psychol. 2012. 41(5): p. 682-94.

20.Hall CA, Reynolds-lii CF. Late-life depression in the primary care setting: challenges, collaborative care, and prevention. Maturitas. 2014. 79(2): p. 147-52. 
21.Zhao Y, Hu Y, Smith JP, et al. Cohort Profile: The China Health and Retirement Longitudinal Study (CHARLS). International Journal of Epidemiology. 2014. 43(1): p. 61-68.

22.http://charls.pku.edu.cn/articles/news/579/zh-cn.html. [accessed 25June 2021].

23.Andresen EM, Malmgren JA, Carter WB, et al. Screening for depression in well older adults: evaluation of a short form of the CES-D (Center for Epidemiologic Studies Depression Scale). Am J Prev Med. 1994. 10(2): p. 77-84.

24.Lee AE, Chokkanathan S. Factor structure of the 10-item CES-D scale among community dwelling older adults in Singapore. Int $J$ Geriatr Psychiatry. 2008. 23(6): p. 592-7.

25.Boey KW. Cross-validation of a short form of the CES-D in Chinese elderly. Int J Geriatr Psychiatry. 1999.14(8):p.608-17.

26.Cheng HG, Chen S, McBride O, et al. Prospective relationship of depressive symptoms, drinking, and tobacco smoking among middleaged and elderly community-dwelling adults: Results from the China Health and Retirement Longitudinal Study (CHARLS). J Affect Disord. 2016. 195: p. 136-43.

27.Slavich, G.M. and M.R. Irwin. From stress to inflammation and major depressive disorder: a social signal transduction theory of depression. Psychol Bull. 2014. 140(3): p. 774-815.

28.Slavich GM, Sacher J. Stress, sex hormones, inflammation, and major depressive disorder: Extending Social Signal Transduction Theory of Depression to account for sex differences in mood disorders. Psychopharmacology (Berl). 2019. 236(10): p. 3063-3079.

29.Colodro-Conde L, Couvy-Duchesne B, Zhu G,et al. A direct test of the diathesis-stress model for depression. Mol Psychiatry. 2018. 23(7): p. 1590-1596.

30.Whinnery J, Jackson N, Rattanaumpawan P, et al. Short and long sleep duration associated with race/ethnicity, sociodemographics, and socioeconomic position. Sleep. 2014.37(3): p.601-11.

31.Li LW, Liu J, Xu H, et al. Understanding Rural-Urban Differences in Depressive Symptoms Among Older Adults in China. J Aging Health. 2016. 28(2): p. 341-62.

32.Hong J, Aspey L, Bao G, et al. Chronic Cutaneous Lupus Erythematosus: Depression Burden and Associated Factors. Am J Clin Dermatol. 2019. 20(3): p. 465-475.

33.Patten SB, Williams JVA, Lavorato DH, et al. Patterns of association of chronic medical conditions and major depression. Epidemiol Psychiatr Sci. 2018. 27(1): p.42-50.

34.Li Y, Wu Y, Zhai L, et al. Longitudinal Association of Sleep Duration with Depressive Symptoms among Middle-aged and Older Chinese. Scientific Reports. 2017.7(1).

35.Gehrman P, Seelig AD, Jacobson IG, et al. Predeployment Sleep Duration and Insomnia Symptoms as Risk Factors for New-Onset Mental Health Disorders Following Military Deployment. Sleep. 2013. 36(7): p. 1009-1018.

36.Luik Al, Zuurbier LA, Direk N, et al. 24-HOUR ACTIVITY RHYTHM AND SLEEP DISTURBANCES IN DEPRESSION AND ANXIETY: A POPULATION-BASED STUDY OF MIDDLE-AGED AND OLDER PERSONS. Depress Anxiety. 2015. 32(9): p. 684-92.

37.Shen J, Barbera J, Shapiro CM. Barbera and C.M. Shapiro. Distinguishing sleepiness and fatigue: focus on definition and measurement. Sleep Med Rev. 2006.10(1): p. 63-76.

38.van Noorden MS, van Fenema EM, van der Wee NJ, et al. Predicting outcome of depression using the depressive symptom profile: the Leiden Routine Outcome Monitoring Study. Depress Anxiety. 2012.29(6):p.523-30.

39.Wang J, Geng L. Effects of Socioeconomic Status on Physical and Psychological Health: Lifestyle as a Mediator. Int J Environ Res Public Health. 2019. 16(2).

40.Ng CW, Tan WS, Gunapal PP, et al. Association of Socioeconomic Status (SES) and Social Support with Depressive Symptoms among the Elderly in Singapore. Ann Acad Med Singap. 2014. 43(12): p. 576-87. 
41.Ouyang P, Sun W. The association between depressive symptoms and fall accidents among middle-aged and elderly people in China. Environ Health Prev Med. 2018 Sep 5;23(1):42.

42.Chan A, Malhotra C, Malhotra R, et al. Living arrangements, social networks and depressive symptoms among older men and women in Singapore. Int J Geriatr Psychiatry.2011.26(6): p.630-9.

43.Kooshiar H, Yahaya N, Hamid TA, et al. Living arrangement and life satisfaction in older Malaysians: the mediating role of social support function. PLoS One. 2012. 7(8): p. e43125.

44. Holtfreter K, Reisig MD, Turanovic JJ. Depression and infrequent participation in social activities among older adults: the moderating role of high-quality familial ties. Aging Ment Health. 2017.21(4):p.379-388.

45.Sander R. Preventing social isolation and loneliness among older people: a systematic review of health promotion interventions. Nurs Older People. 2005. 17(1): p. 40.

46.Kendler KS, Hettema JM, Butera F, et al. Life event dimensions of loss, humiliation, entrapment, and danger in the prediction of onsets of major depression and generalized anxiety. Arch Gen Psychiatry. 2003. 60(8): p. 789-96.

47.Lorant V, Deliège D, Eaton W, et al. Socioeconomic inequalities in depression: a meta-analysis. Am J Epidemiol. 2003. 157(2): p. 98-112.

48. Hosseinpoor AR, Bergen N, Mendis S, et al. Socioeconomic inequality in the prevalence of non-communicable diseases in low-and middle-income countries: results from the World Health Survey. BMC Public Health. 2012. 12: p. 474.

49.Martel MM. Sexual selection and sex differences in the prevalence of childhood externalizing and adolescent internalizing disorders. Psychol Bull. 2013. 139(6): p. 1221-59.

50.Chen R, Copeland JR, Wei L. Copeland and L. Wei. A meta-analysis of epidemiological studies in depression of older people in the People's Republic of China. Int J Geriatr Psychiatry. 1999. 14(10): p.821-30.

51.He X, Song M, Qu J, et al. Basic and translational aging research in China: present and future. Protein Cell. 2019. 10(7): p.476-484.

52.Gong F, Zhao D, Zhao Y, et al. The factors associated with geriatric depression in rural China: stratified by household structure. Psychol Health Med. 2018. 23(5): p. 593-603.

53.Fang EF, Scheibye-Knudsen M, Jahn HJ,et al. A research agenda for aging in China in the 21st century. Ageing Res Rev. 2015. 24(Pt B): p. 197-205. 